\title{
Phage-Chips for Novel Optically Readable Tissue Engineering Assays
}

\author{
So Young Yoo, ${ }^{\dagger}$ Jin-Woo Oh, ${ }^{\dagger}$ and Seung-Wuk Lee*
}

Department of Bioengineering, and Berkeley Nanoscience and Nanoengineering Institute, University of California, Berkeley, Berkeley, California 94720, United States

Physical Biosciences Division, Lawrence Berkeley National Laboratory, Berkeley, California 94720, United States

\section{Supporting Information}

ABSTRACT: We report novel phage-based array chips that are optically readable for cell proliferation and morphology assays. Using M13 phages that were engineered to display RGD on its major coat proteins and/or immobilize $\mathrm{FGFb}$ on its minor coat proteins, we prepared arrays of phage spot matrices composed of self-assembled nanofibrous network structures. We cultured fibroblasts on the arrays and, using surface plasmon resonance (SPR) spectroscopy, monitored the effects of the biochemical cues displayed by the phage on cell proliferation and morphology. This study demonstrates the utility of engineered phages as promising coating materials for lab-on-a-chip (LOC) platforms, allowing sensitive monitoring of the effects of functional peptides on cell growth. Phage-chips have great potential for use as high-throughput screening systems for biochemical assays and
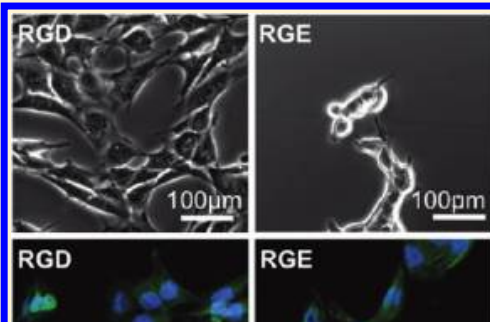

Wild

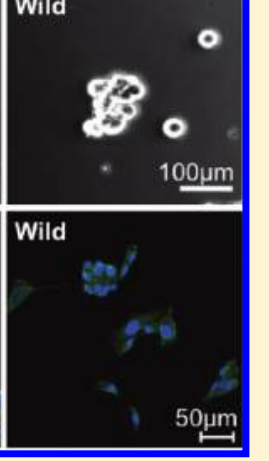
biosensors and the discovery of novel drugs.

\section{INTRODUCTION}

Precise control of the structural organization of regenerative tissue materials is critical for mimicking the natural tissue microenvironment.$^{1-7}$ Biomimetic materials have been synthesized using top-down and bottom-up approaches to approximate the level of complexity observed in the natural extracellular environment. ${ }^{6,8-14}$ Previous studies have shown that chemical cues (i.e., signaling peptides, growth factors, cytokines), physical cues (fiber dimension, porosity, topography), and mechanical stiffness are critical for inducing desired tissue structures and morphologies. ${ }^{15-17}$ Genetically engineered phages have recently been shown to have great potential for use in developing novel tissue regenerative materials. ${ }^{18-23} \mathrm{~A}$ phage has several attributes (i.e., monodispersity, nanofibrous shape, and versatility in displaying multiple signaling peptides on their coat proteins) that make them ideal for use in developing soft tissue engineering materials. ${ }^{24-26}$ We have previously demonstrated that phage-based tissue engineering matrices are good model systems for investigating biochemical and physical cues. Here, we report the use of a genetically engineered phage in optically readable phage-chip arrays for cell proliferation and morphology assays. We utilized previously developed M13 phages that were engineered to display integrinbinding peptide (RGD) or the biotin-like peptide (HPQ) for conjugating growth factors. We then prepared arrays of selfassembled phage matrices and characterized the growth of fibroblasts (NIH3T3) on these arrays using surface plasmon resonance (SPR) spectroscopy. We demonstrated that these phage-chip arrays can be used as novel and convenient toolkits for quantifying changes in cell growth and morphology in tissue engineering.

\section{EXPERIMENTAL SECTION}

Genetic Engineering and Purification of M13 Bacteriophage. An inverse polymerase chain reaction (PCR) cloning method was used to insert peptide motifs on every copy of the M13 major (pVIII) and minor (pIII) coat proteins. ${ }^{27,28}$ Phages were constructed to present GRGDS motifs with DDY amino acids at the N-terminus of every copy of pVIII using previously described inverse PCR methods ${ }^{25}$ (see Table 1 for primer sequences). For pIII peptide display, a slightly altered approach was used. The peptide motifs were inserted immediately at the N-terminus of pIII. An M13KE phage vector (New England Biolabs, Ipswich, MA), which had an engineered EagI restriction enzyme site at the $\mathrm{N}$-terminus of the mature PIII protein, was used, along with a pIII reverse primer that was designed to include the EagI restriction site, the insert sequence, and a segment complementary to the gIII 5 '-3' strand. A pIII forward primer was designed to make the vector linear and was fully complementary to the engineered gIII 3'-5' region, which included an EagI restriction site (see Table 1 for primer sequences). To incorporate the gene sequences, PCR was performed using Phusion High-Fidelity DNA polymerase, the two primers, and the M13KE vector, either as supplied or previously engineered at $\mathrm{pVIII}$. The obtained product was purified on an agarose gel, eluted by spin column purification, digested with Eag I enzyme (New England Biolabs), and recircularized overnight at $16{ }^{\circ} \mathrm{C}$ with T4 DNA Ligase (New England Biolabs). ${ }^{29}$ The ligated DNA vector was then transformed into XL10-Gold Ultracompetent bacteria cells (Stratagene, La Jolla, CA), and the amplified plasmid was

Special Issue: Bioinspired Assemblies and Interfaces

Received: September 30, 2011

Revised: November 29, 2011 
Table 2. Genetically Engineered Functionalized pVIII and pIII Phage ${ }^{a}$

\begin{tabular}{|c|c|c|c|}
\hline name & PVIII & PIII & reference \\
\hline $\mathrm{RGD}_{8}$ & $\mathrm{ADSGRGDTEDP}$ & SHSAET & $\begin{array}{l}\text { Merzlyak et al.; }{ }^{25} \\
\text { Chung et al. }{ }^{24} \text { Yoo } \\
\text { et al. }{ }^{21-23}\end{array}$ \\
\hline $\mathrm{RGE}_{8}$ & ADSGRGETEDP & SHSAET & $\begin{array}{l}\text { Chung et al.; }{ }^{24} \text { Yoo } \\
\text { et al. }\end{array}$ \\
\hline wild-type & AEGDDP & SHSAET & $\begin{array}{l}\text { purchased from New } \\
\text { England Biolabs } \\
\text { (Ipswich, MA) }\end{array}$ \\
\hline $\begin{array}{l}\text { GRGDSDDY }_{8} \\
\mathrm{HPQ}_{3}\end{array}$ & $\mathrm{AGGRGDSDDY \textrm {DP }}$ & $\begin{array}{l}\text { SHSA-C- } \\
\text { HPQGPL-C- } \\
\text { GGGSAET }\end{array}$ & this study \\
\hline
\end{tabular}

${ }^{a}$ Peptide inserts are shown in italics. Functional sequences are also underlined.

verified via DNA sequencing at the UC Berkeley DNA Sequencing

Facility (Berkeley, CA).

Fabrication of Phage-Chip. To make a uniform phage film on substrates, we constructed a home-built phage deposition apparatus by modifying a syringe pump motor (KD Scientific Inc., Holliston, MA). Gold coated glass (Platypus Tech, Madison, WI) was treated with $3 \mathrm{mM}$ cysteamine in deionized (DI) water for $30 \mathrm{~min}$ (to increase adhesion of the phage to the glass) and then washed by DI water and ethanol. Phages were then applied to the treated glass at a concentration of $3.0 \mathrm{mg} / \mathrm{mL}$ by a pulling up method. ${ }^{22}$ To make arrays of phage drop cast films (Figure 1. Inset), phage solutions $\left(\sim 10^{12}\right.$ phages $/ \mathrm{mL}$ in phosphate buffered saline (PBS)) were drop-cast on the gold-coated glass and allowed to dry overnight at room temperature. The deposited phage films were then cross-linked in a sealed chamber filled with glutaraldehyde vapor (3 days) to prevent them from peeling off in the culture media. Any remaining aldehydes were blocked with 2-aminoethanol solution $(100 \mathrm{mM})$ for $30 \mathrm{~min}$.

SPR Optical System. SPR spectra were characterized using on the Kretschmann optical configuration. Figure 1 shows the experimental arrangement of SPR setup used in this work. A tungsten halogen lamp with a multiwavelength light source was used, and a polarizer was positioned on the input path of the light for transverse magnetic fields. The prism coupler and the phage-chip were mounted on three linearmotion stages to adjust the $x-y-z$ position. The reflected spectrum was measured by a fiber optic spectrometer (USB4000-UV-vis, Ocean Optics, Dunedin, FL), and data acquisition was performed using the Spectra Suite software. The SPR spectrum was calculated from linearly polarized light parallel/perpendicular to the incidence plane (TM/TE configuration).

Mouse Fibroblast Cell Culture. NIH3T3 mouse fibroblast cells were obtained from Cell Facilities at the University of California, Berkley (Berkeley, CA) and used at passages 20-30. Cells were cultured in Dulbecco's modified Eagle's medium (DMEM) medium (Invitrogen, Carlsbad, CA) supplemented with $10 \%$ fetal bovine serum (FBS) and $1 \%$ antibiotics-antimicotics at $37{ }^{\circ} \mathrm{C}$ in $5 \% \mathrm{CO}_{2}$ under humidified conditions. The growth media was changed every 2 days, and cells were subcultured using trypsin/EDTA near confluency.

Streptavidin Conjugation of FGFb. The Lightning-Link conjugation kit (Innova Biosciences, Cambridge, U.K.) was used to conjugate $\mathrm{FGFb}$ to streptavidin. The amine group of FGFb was activated by adding LL-modifier reagent. Incubation with the Lightning-Link mixture resulted in coupling of the FGFb to streptavidin.

WST-1 Cell Proliferation Assay. The WST-1 assay (Cell Proliferation Reagent WST-1, Roche Applied Science, Basel, Switzerland) was performed as per manufacturer's instructions. Ten microliters of reagent were mixed with $0.1 \mathrm{~mL}$ of growth medium and added to the NIH3T3 cells grown on $\mathrm{RGD}_{8}$-phage and other control matrices. The difference between the absorbances at 450 and $690 \mathrm{~nm}$ of the sample was read on an ELISA reader (Safire, Tecan Group Ltd., Mannedorf, Switzerland).

Immunostaining and Data Analysis. Cells were fixed in 3.7\% formaldehyde solution for $15 \mathrm{~min}$ and then blocked with a solution 


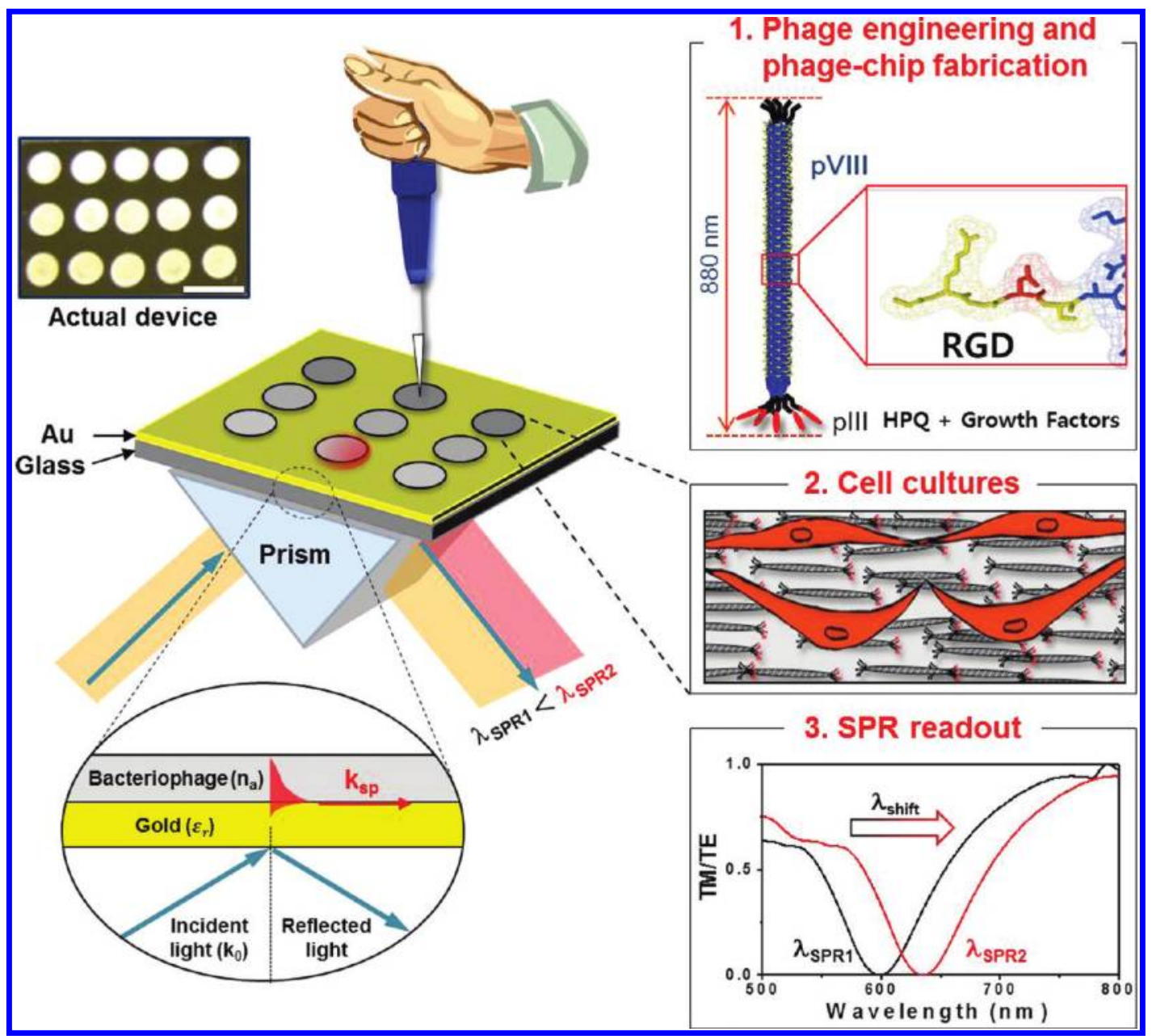

Figure 1. Photograph and schematic diagram of phage-SPR chip for monitoring cell proliferation. Photograph of phage-SPR chip (scale bar represents $1 \mathrm{~cm}$ ). (1) Genetic engineering of major coat proteins of M13 phages with RGD (or RGE) peptide and minor coat proteins with HPQ peptide. The engineered phages were then used for coating the surface of the SPR-chip. (2) Cell proliferation on the phage-chip. (3) In SPR analysis, cells respond differently according to the peptides presented by phage coat proteins.

of $0.3 \%$ Triton X-100 and 5\% normal goat serum in $1 \mathrm{X}$ PBS for 30 min. Primary antibodies for identifying cell markers and for M13 phage were incubated with the cells overnight at $4{ }^{\circ} \mathrm{C}$. The primary antibodies used in this investigation were mouse anti- $\beta$-tubulin III antibody (1:400, Sigma Aldrich, St. Louis, MO) and rabbit anti-fd antibody (1:500, Sigma Aldrich, St. Louis, MO). Secondary goat Alexa flourochrome-conjugated antibodies (Molecular Probes, Eugene, OR) and/or actin phalloidin were used at a dilution of 1:250 to visualize the markers and incubated with the cells for $2 \mathrm{~h}$ at room temperature. A DAPI (Molecular Probes, Eugene, OR) solution in PBS was used as a nuclear counterstain for all samples.

\section{RESULTS AND DISCUSSION}

Construction of the Phage-Array SPR Chip. We constructed phage-chip arrays comprised of self-assembled phage that were engineered to display various chemical cues. We had previously constructed M13 phage to display integrin-binding peptide (RGD) on its major coat proteins $\left(\mathrm{RGD}_{8}\right.$-phage) and demonstrated that it could induce specific focal adhesions and stimulate cells to migrate and proliferate throughout selfaligned nanofibrous phage tissue networks. ${ }^{21,24,25}$ We also demonstrated that the $\mathrm{RGD}_{8}$-phage could be modified to display an additional biotin-like peptide (HPQ peptide) on its minor coat protein $\left(\mathrm{RGD}_{8}-\mathrm{HPQ}_{3}\right.$ phage) to allow it to be conjugated to various growth factors for regulating cell growth. ${ }^{22}$ We used these $\mathrm{RGD}_{8^{-}}$and $\mathrm{RGD}_{8}-\mathrm{HPQ}_{3}$-phages to fabricate arrays of phage on gold-coated glass substrates. To enhance the interaction between the phage and the gold substrate, we further modified the $\mathrm{RGD}_{8}$-phage to include two aspartate (DD) motifs and coated the gold substrates with cysteamines through self-assembled monolayer (SAM) formation.

Each "phage-chip" consisted of an array of phages as coating materials for an SPR-based monitoring (Figure 1). $\mathrm{RGD}_{8}$, $\mathrm{RGE}_{8}$-phage, wild type-phage (displaying no peptide), or $\mathrm{RGD}_{8}-\mathrm{HPQ}_{3}$-phage bound to streptavidin-conjugated growth factors, FGFb (strep-FGFb) were utilized as coating materials. The interactions between the phage-matrices and cells grown on top of them were monitored through SPR spectroscopy. The SPR optical characteristics (i.e., reflective angles, peak wavelengths) in the phage-chip, were influenced by the interactions between the cells and the coated phages. Reference readings under control conditions were used to calibrate the signals. SPR spectra were measured in situ in a time lapsed manner. Wavelength shifts $\left(\lambda_{\text {SPR } 2}-\lambda_{\text {SPR1 }}\right)$ were used to monitor cell number changes (Figure 1).

SPR Responses of Phage-Modified Chips for System Verification. The phage-chips exhibited concentration-dependent SPR wavelength shifts. Figure 2 shows spectrum shift profiles when samples at different concentrations of cysteamine and phage were coated onto the SPR chip. The signals obtained from the interactions between the target molecules and surfaces 


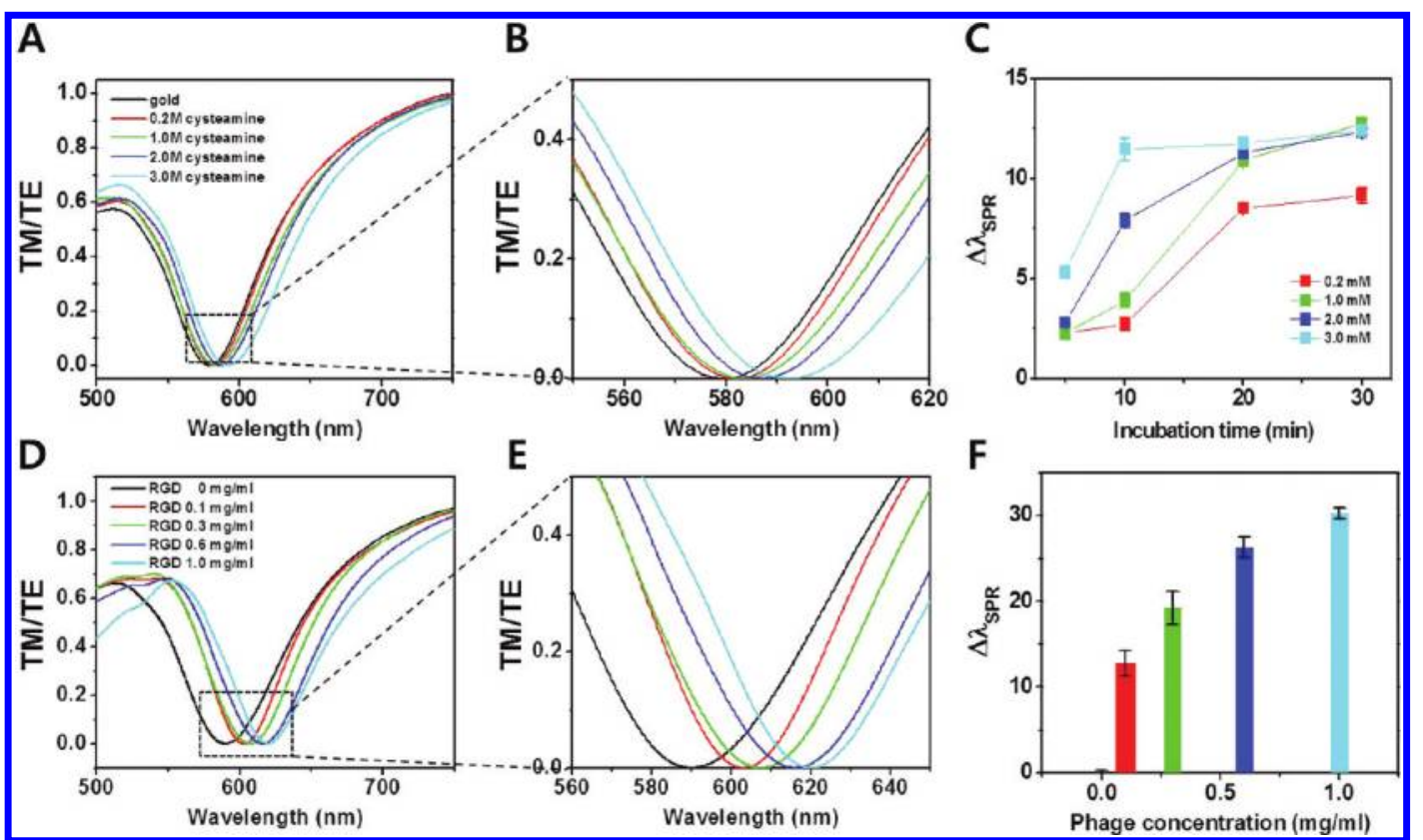

Figure 2. SPR spectrum profiles and calibration plots of the phage-chips. The concentration of cysteamine applied onto the Au surface was varied from 0 to $3 \mathrm{mM}$ for $10 \mathrm{~min}(\mathrm{~A}, \mathrm{~B})$. SPR wavelength shift upon the application of different concentrations of cysteamines for $0-30 \mathrm{~min}$ incubation time (C). The concentration of phages applied onto the cysteamine surface was varied from 0.1 to $1 \mathrm{mg} / \mathrm{mL}$ (D,F). The shift of SPR wavelength increased with concentrations of deposited materials.

materials on the chip depended on parameters such as incubation time, type of coating materials, concentrations of target molecules, and/or ionic strength, which requires optimization. To stabilize the phage coating on a gold-coated glass substrate, we deposited a SAM of cysteamine on the chip. To investigate the effect of cysteamine and phage on the SPR phenomenon, we first obtained the SPR data with different concentrations of cysteamine and phage, respectively. The spectrum profile obtained from the bare Au surface showed a SPR wavelength at $\sim 582 \mathrm{~nm}$ (black line) (Figure $2 \mathrm{~A}-\mathrm{C}$ ). When $0.2 \mathrm{mM}$ cysteamine was immobilized on the surface of the SPR chip for $10 \mathrm{~min}$, we observed a shift of the SPR peak to a higher wavelength (red line in Figure 2A,B). The SPR peak signal became more red-shifted as the concentration of the cysteamines increased. The calibration plots shown in Figure 2C display the dependence of the SPR wavelength shifts on the concentrations of target molecules (cysteamine) depending on the incubation time. The SPR signal (wavelength) detecting cysteamine surface coverage was saturated with increasing the cysteamine solution in the range of 1-3 $\mathrm{mM}$ within $30 \mathrm{~min}$ incubation time (Figure 2C).

We examined the effect of phage concentration on the SPR spectrum profiles using chips deposited with $3.0 \mathrm{mM}$ cysteamine, (Figure $2 \mathrm{D}-\mathrm{F}$ ). With $0.1 \mathrm{mg} / \mathrm{mL}$ phage, the SPR peak was observed at $\sim 608 \mathrm{~nm}$ (black line). The SPR signal became also more red-shifted as phage concentration increased $(0.3$, 0.6 , and $1 \mathrm{mg} / \mathrm{mL})$. The calibration plots of figure $2 \mathrm{~F}$ display the dependence of the SPR wavelength shift on phage concentration. SPR wavelength data with increasing concentrations of phage solutions, however, did not reach the saturation point. This may be because the different thickness made by phages strongly affects the SPR signal since the SPR wavelength shifted to a longer wavelength with increasing sample thickness (Figure S1). ${ }^{30-33}$ The standard deviations of the values were less than $10 \%$ in all the measurements, indicating that theses phage-chips were fabricated uniformly and would be suitable for use in future sensing applications.
Quantification of Cell Numbers Using the Phage-SPR Chip. We can quantify cell numbers using the cysteamine-chip through SPR analysis. The effects of different numbers of NIH3T3 mouse fibroblast cells applied onto cysteamine SAMSPR-chips are shown in Figure 3A-C. Cells in culture media were applied onto the chip and then washed with PBS after a $2 \mathrm{~h}$ incubation. This amount of time was expected for cell attachment without proliferation. Normally, the maximum number of cells per square centimeter is $1.4-3.6 \times 10^{5}$ cells $/ \mathrm{cm}^{2}$ graft, for adherent cell types. ${ }^{34}$ Since the light source passes through the $\sim 3 \mathrm{~mm}^{2}$ surface area in our SPR system, we would not able to get a clear SPR peak with over 5000 cells. Thus, different numbers $(0,20,40,160,320,640$ and 1280) of cells were applied and analyzed by SPR spectroscopy. Fewer than $10^{4}$ cells cannot be detected by conventional assays such as WST-1 (Figure 3D). Figure 3A,B shows the SPR spectrum profiles of different numbers of cells applied onto the chip. As expected, the SPR wavelength became more red-shifted as the numbers of cells increased. Without applying the NIH3T3 cell, the spectrum profile obtained from the cysteamine-SPR-chip showed a SPR wavelength of $\sim 598 \mathrm{~nm}$ (black line) (Figure $3 \mathrm{~A}, \mathrm{~B})$. The calibration plots of Figure $3 \mathrm{C}$ show the dependence of the SPR wavelength shift $\left(\Delta \lambda_{\text {SPR }}\right)$ on the numbers of target molecules (cells). SPR shift occurred accordingly with increasing number of cells, between 20 and 1280 cells. Therefore, our rapid and sensitive SPR system can be used for cell counting and proliferation assays as well as for chemical target recognition such as protein/viruses.

Cellular Proliferation Monitoring Using Phage-SPR Chips. We monitored cellular proliferation using phage-chips adapted with engineered phage to display integrin-binding peptide RGD, a control RGE peptide, or no peptide (wild-type phage). We then examined the effects of these phage-chips on the growth of mouse fibroblast cells (NIH3T3). Previously, we showed that phage engineered to display bioactive peptides on the 2700 copies of their major coat proteins could effectively 


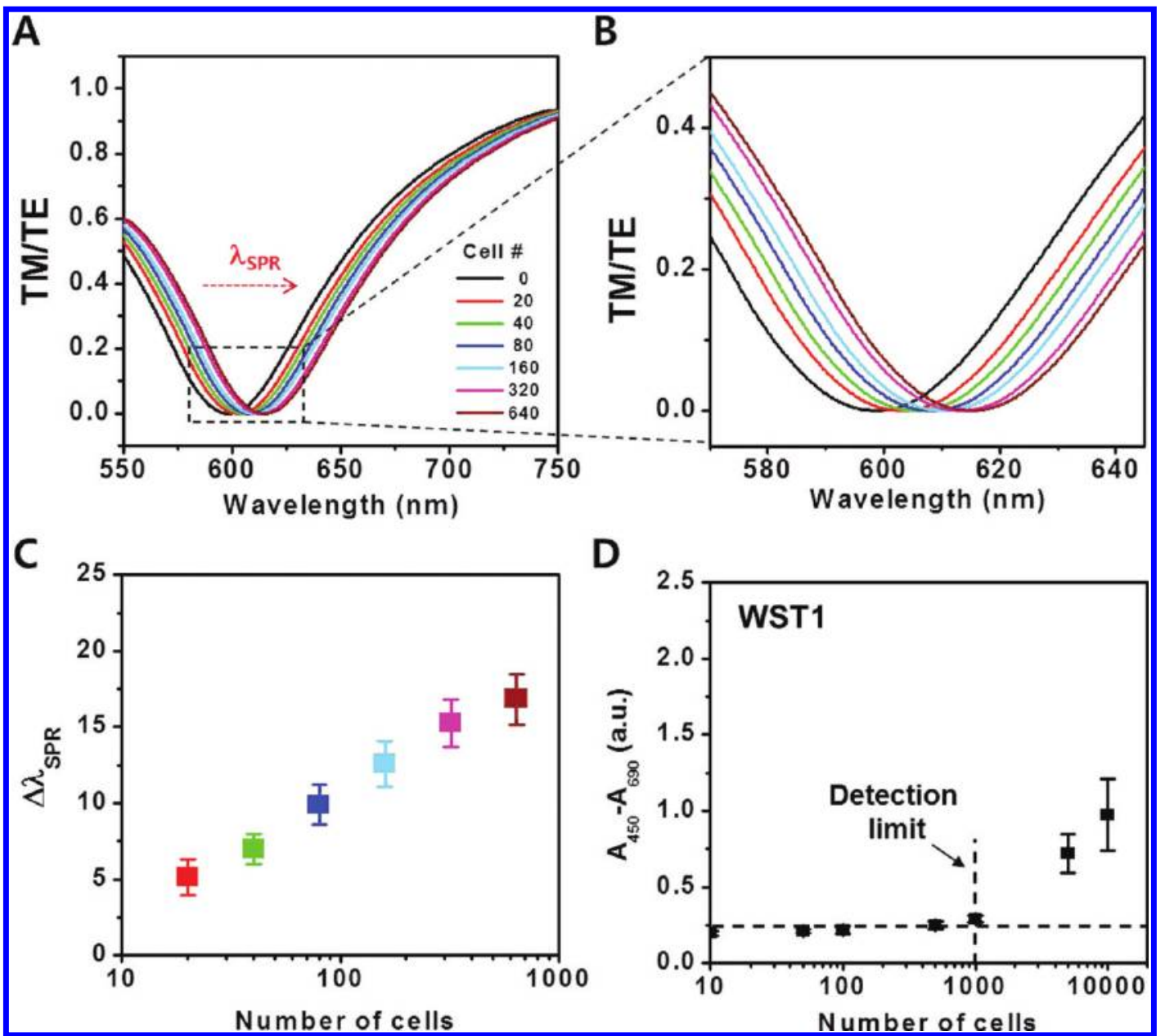

Figure 3. SPR spectrum profiles and WST-1 assay results obtained using different numbers of cells. Superimposed spectrum curves and calibration plots obtained after applying cells on cysteamine treated gold (Au) surfaces $(\mathrm{A}-\mathrm{C})$. The numbers of the target cells were varied from 20 to 640 , a range that cannot be detected by the conventional WST-1 assay (D). As the number of cells increased, the SPR wavelength shift $\left(\Delta \lambda_{\mathrm{SPR}}\right)$ increased. Results of the WST-1 assay using 10 to $10^{4}$ cells showed that this assay can be used only when cell numbers over $10^{4}$ cells are used. The signal at zero cells indicates that of media only.

provide biochemical, physical, and mechanical cues to surrounding cells. The advantages of using these phages as biosensors and tissue engineering materials were also previously discussed. $^{21,22}$ When the effects of SAM of RGD-peptide and $\mathrm{RGD}_{8}$-phage were compared in controlling cellular direction and morphology, the $\mathrm{RGD}_{8}$-phage SAM was found to be much more effective in guiding cell growth than the RGD-peptide SAM alone. In this study, we used SPR analyses to examine the effects of the three engineered phages on the proliferation of NIH3T3 cells at $0,2,4,8,12,24,48$, and 72 h of culture. Cells were initially plated at $10^{3}$ cells. We observed that cells on the $\mathrm{RGD}_{8}$-phage sample exhibited greater SPR signal shifts than those of the $\mathrm{RGE}_{8^{-}}$or wild-type- phages. Interestingly, this difference was observed even at 2, 4, 8, and $12 \mathrm{~h}$. Since most cellular doubling times are much longer than $12 \mathrm{~h}$, our SPR spectrum profile shifts may reflect cell morphological responses to biochemical cues provided by the engineered phages before cell proliferation occurred because the SPR spectrum might reflect surface coverage or cellular area. The different morphological cellular responses to $\mathrm{RGD}_{8^{-}}, \mathrm{RGE}_{8^{-}}$or wild-type phages at early time points have been previously discussed. ${ }^{20,35}$ Also because adhesion force given by RGD peptide on phages to cells allows more cellular attachment when plating, it might cause greater SPR signal shifts even before $12 \mathrm{~h}$. Cellular proliferation was detected by SPR (Figure 4A) and visualized by immunofluorescence (IF) (Figure 4B). Our results show not only that the cells responded specifically to the RGD provided by the phage, but also that the selectivity and sensitivity of the phage-SPR chip were comparable to those of conventional assay systems.

Cell Proliferation Assays Triggered by Growth Factors Immobilized On Phage-SPR Chips. We monitored cell proliferation triggered by different concentrations of growth factors immobilized on the phage-chips. We constructed $\mathrm{RGD}_{8^{-}}$ $\mathrm{HPQ}_{3}$-phages to display integrin-binding (RGD) peptides on their major coat proteins and biotin-like (HPQ) peptides on their minor coat proteins. These multifunctional phages easily bound to several streptavidin-conjugated growth factors, regardless of their size, and were able to stimulate and guide cells to proliferate with desired morphologies. The effects of the phages on cell proliferation were examined using our phage-chip system and a WST-1 assay (Figure 5). Because the detectable range of cell numbers was limited in the WST-1 assay system, we plated a higher numbers of cells $\left(10^{4}\right)$ for the WST-1 assay at day 0 and only $10^{3}$ cells for the SPR-based assay. Prior to cell plating, we coated the phage-SPR chip with $1 \mathrm{mg} / \mathrm{mL} \mathrm{RGD}_{8}-\mathrm{HPQ}_{3}$-phage and then linked the phage with $400 \mathrm{ng} / \mathrm{mL}$ strep-FGFb. These concentrations of phages and strep-FGFb were determined after optimization. Cells were then seeded onto the phage-SPR chips 


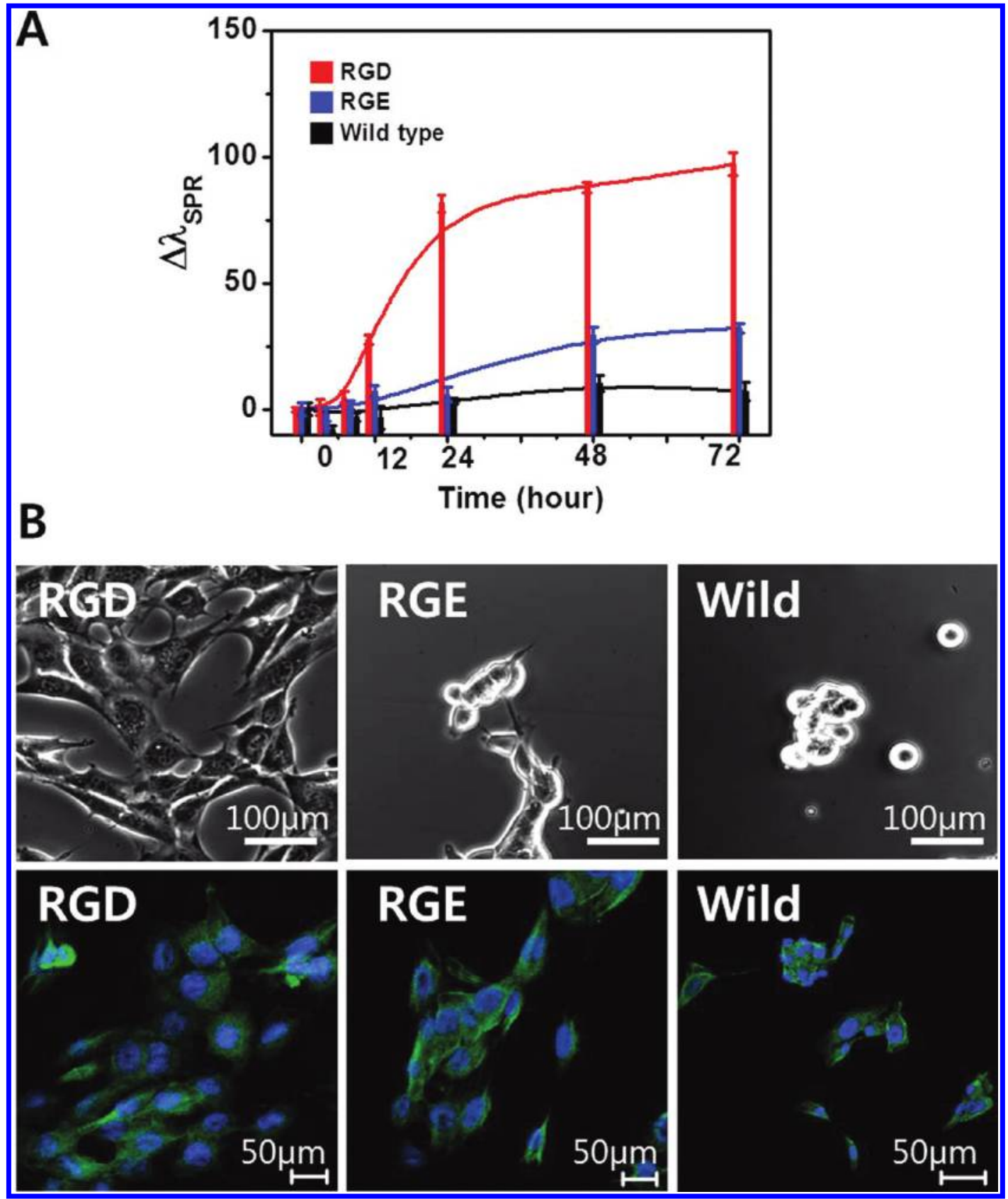

Figure 4. Monitoring of NIH3T3 proliferation using phage-SPR chips. (A) SPR data plot showing cell proliferation in the presence of $\mathrm{RGD}_{8^{-}}$, $\mathrm{RGE}_{8^{-}}$ or wild-type phage. SPR signals recorded between 2 and $12 \mathrm{~h}$ show that SPR can be used to monitor not only cell proliferation (cell number) but also cell morphology (cell area). (B) Microscopic and immunostaining images of mouse fibroblast cells (NIH3T3) grown on $\mathrm{RGD}_{8^{-}}$, RGE $\mathrm{R}^{-}$or wildtype (unmodified) phages. (green: mouse beta-tubulin III; blue: DAPI, nuclei; images were taken after $48 \mathrm{~h}$ culture).

and cultured for 3 days in DMEM containing 2\% FBS media instead of DMEM containing 10\% FBS media. The lowered FBS concentration was used to initially synchronize cell proliferation, diminish growth effects stemming from the media, and allow us to observe only the effects of the phages on the cells. At different time points, the chips were removed from the incubator, and their cell proliferation rates were assayed by SPR analysis and WST-1, respectively (Figure 5A,B). We observed that cells grown on $\mathrm{RGD}_{8}-\mathrm{HPQ}_{3}$-phage in the presence of strep-FGFb showed a higher degree of proliferation than those on $\mathrm{RGD}_{8}-\mathrm{HPQ}_{3}$-phages in the absence of strep-FGFb in cases of using both SPR-based phage-chip assay and WST1 assay. The rates of proliferation in the presence of strep-FGFb increased with culture time. The effects of increasing concentrations of strep-
$\mathrm{FGFb}$ were examined, confirming that the cell proliferation increase was due to the presence of FGFb immobilized onto the phage-chip (Figure 5C).

This study demonstrated the use of a rapid, selective, and sensitive SPR based phage-chip system to perform cellular assays. We used engineered phages that displayed bioactive peptides as coating materials for controlling target molecular responses. Our multifunctional phages provide a means for presenting biochemical and physical cues, the effects of which can be easily measured by SPR spectroscopy. Although sensitivity of the phage-chips might be limited once it is fully covered by the target cells at maximum cell density, our phagechip for monitoring cell proliferation and morphology is rapid, very sensitive, and comparable to convention cellular assays. 


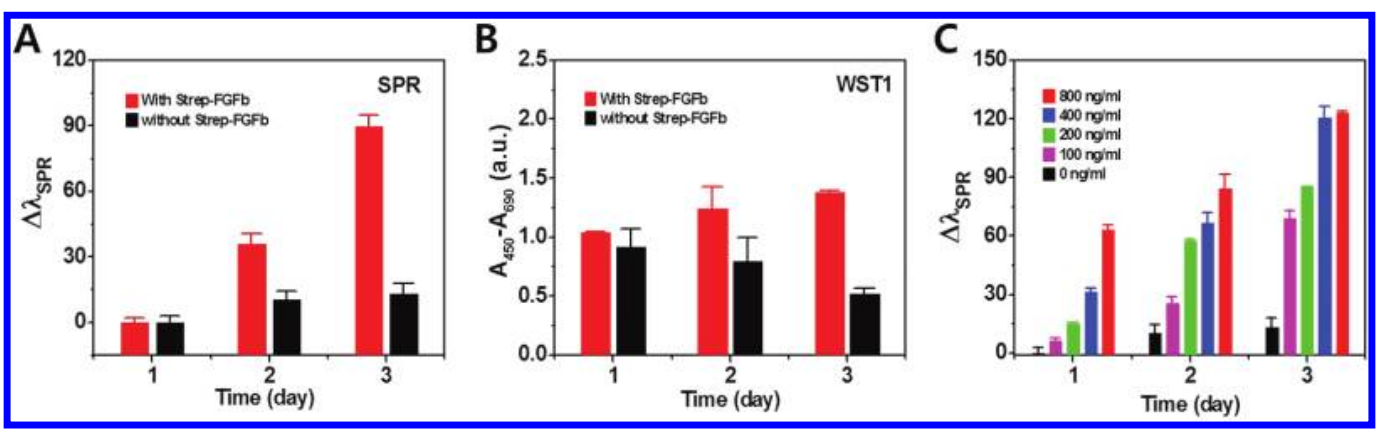

Figure 5. Cell proliferation assays used to monitor the effects of growth factor immobilized by $\mathrm{RGD}_{8}$ - $\mathrm{HPQ}_{3}$-phage-SPR data (A) and WST-1 assay (B). Cells at 1000 cells were cultured with or without strep-FGFb $(400 \mathrm{ng} / \mathrm{mL})$. The HPQ peptides of $\mathrm{RGD}_{8}-\mathrm{HPQ}_{3}$ were used to immobilize streptavidin-conjugated FGFb onto the phages. (C) Increasing concentrations of strep-FGFb induced greater cell proliferation, which could be successfully measured by SPR.

\section{CONCLUSIONS}

We developed a facile and sensitive label-free SPR-based cell proliferation assay chip employing engineered phages as novel coating materials. The chip is easy to use, provides rapid results, and can detect fewer than 1000 cells. This SPR based phage-chip successfully detected cell responses to physical and chemical cues presented by RGD-, RGE- or wild-type phage and phageconjugated FGFb. To our knowledge, this is the first documentation to report an SPR-based cell chip to monitor cell proliferation using engineered phages as a coating material. This phage-chip-based proliferation assay provides a new and convenient platform for investigating several parameters of cell biological functions.

\section{ASSOCIATED CONTENT}

\section{S Supporting Information}

The relationship between the film thickness controlled by phage concentration and the SPR wavelength shift $\left(\Delta \lambda_{\mathrm{SPR}}\right)$. This material is available free of charge via the Internet at http://pubs.acs.org.

\section{AUTHOR INFORMATION}

\section{Corresponding Author}

*E-mail: leesw@berkeley.edu.

\section{Author Contributions}

${ }^{\dagger}$ These authors contributed equally to this work.

\section{ACKNOWLEDGMENTS}

This work was supported by the Hellman Family Faculty Fund, start-up funds from the Berkeley Nanoscience and Nanoengineering Institute at the University of California, Berkeley, and the Laboratory Directed Research and Development fund from the Lawrence Berkeley National Laboratory.

\section{REFERENCES}

(1) Bearinger, J. P.; Terrettaz, S.; Michel, R.; Tirelli, N.; Vogel, H.; Textor, M.; Hubbell, J. A. Nat. Mater. 2003, 2, 259.

(2) Falconnet, D.; Csucs, G.; Michelle Grandin, H.; Textor, $\underline{\text { M. }}$ Biomaterials 2006, 27, 3044.

(3) Lussi, J. W.; Falconnet, D.; Hubbell, J. A.; Textor, M.; Csucs, G. Biomaterials 2006, 27, 2534.

(4) Lussi, J. W.; Csucs, G.; Textor, M.; Hubbell, J. A.; Danuser, G. Mol. Biol. Cell 2002, 13, 1898.

(5) Qin, D.; Xia, Y. N.; Whitesides, G. M. Nat. Protoc. 2010, 5, 491.

(6) Stevens, M. M.; Mayer, M.; Anderson, D. G.; Weibel, D. B.; Whitesides, G. M.; Langer, R. Biomaterials 2005, 26, 7636.

(7) Xia, Y.; Whitesides, G. M. Annu. Rev. Mater. Sci. 1998, 28, 153.
(8) Engel, E.; Michiardi, A.; Navarro, M.; Lacroix, D.; Planell, J. A Trends Biotechnol. 2008, 26, 39.

(9) Langer, R; Vacanti, J. Science 1993, 260, 920.

(10) Schense, J. C.; Bloch, J.; Aebischer, P.; Hubbell, J. A. Nat. Biotechnol. 2000, 18, 415.

(11) Silva, G. A.; Czeisler, C.; Niece, K. L.; Beniash, E.; Harrington, D. A.; Kessler, J. A.; Stupp, S. I. Science 2004, 303, 1352.

(12) Yang, F.; Murugan, R.; Wang, S.; Ramakrishna, S. Biomaterials 2005, 26, 2603.

(13) Teng, Y. D.; Lavik, E. B.; Qu, X.; Park, K. I.; Ourednik, J.; Zurakowski, D.; Langer, R.; Snyder, E. Y. Proc. Natl. Acad. Sci. 2002, 99, 3024.

(14) Zhang, S. Nat. Biotechnol. 2003, 21, 1171.

(15) Yang, S.; Leong, K.-F.; Du, Z.; Chua, C.-K. Tissue Eng. 2001, 7,679 .

(16) Liao, S.; Chan, C. K.; Ramakrishna, S. Mater. Sci. Eng. C 2008, $28,1189$.

(17) Ghosh, K.; Ingber, D. E. Adv. Drug Deliverv Rev. 2007, 59, 1306.

(18) Lee, S. W.; Lee, S.; Belcher, A. Adv. Mater. 2003, 15, 689.

(19) Merzlyak, A.; Indrakanti, S.; Lee, S. W. Nano Lett. 2009, 9, 846.

(20) Chung, W.-J.; Merzlyak, A.; Yoo, S. Y.; Lee, S.-W. Langmuir 2010, 26, 9885 .

(21) Yoo, S. Y.; Chung, W. J.; Kim, T. H.; Le, M.; Lee, S. W. Soft Matter 2011, 7, 363.

(22) Yoo, S. Y.; Merzlyak, A.; Lee, S.-W. Soft Matter 2011, 7, 1660.

(23) Yoo, S. Y.; Kobayashi, M.; Lee, P. P.; Lee, S.-W. Biomacromolecules 2011, 12, 987.

(24) Chung, W. J.; Merzlyak, A.; Yoo, S. Y.; Lee, S. W. Langmuir 2010, 26, 9885.

(25) Merzlyak, A.; Indrakanti, S.; Lee, S. W. Nano Lett. 2009, 9, 846.

(26) Chung, W. J.; Merzlyak, A.; Lee, S. W. Soft Matter 2010, 6, 4454

(27) Chen, G.; Courey, A. J. BioTechniques 1999, 26, 814.

(28) Qi, D.; Scholthof, K. B. L. Virol. Methods 2008, 149, 85.

(29) Sambrook, J.; Russell, D. W. Molecular Cloning: A Laboratory Manual, 3rd ed.; CSHL Press: Woodbury, NY, 2001.

(30) Okamoto, T.; Yamaguchi, I.; Kobayashi, T. Opt. Lett. 2000, $25,372$.

(31) Andersson, O.; Larsson, A.; Ekblad, T.; Liedberg, B. Biomacromolecules 2008, 10, 142.

(32) Otsuki, S.; Tamada, K.; Wakida, S. Appl. Opt. 2005, 44, 3468

(33) Chung, W.-J.; Oh, J.-W.; Kwak, K.; Lee, B. Y.; Meyer, J.; Wang, E.; Hexemer, A.; Lee, S.-W. Nature 2011, 478, 364.

(34) Schneider, P. A.; Hanson, S. R.; Price, T. M.; Harker, L. A. Surgery 1988, 103, 456.

(35) Chung, W.-J.; Merzlyak, A.; Lee, S.-W. Soft Matter 2010, 6, 4454. 